\title{
Modeling of the high-performance PLD-based sectioning method for classification of the shape of optical object images
}

\author{
Leonid Tymchenko ${ }^{1 *}$, Mycola Petrovskiy ${ }^{1}$, Natalia Kokryatskaya ${ }^{1}$, Volodymir Gubernatorov ${ }^{1}$ and Yuriy Kutaev ${ }^{2}$
}

\begin{abstract}
An analysis of the sectioning method to control a shape of the laser beam spot is conducted in this paper. A possibility is discussed to use a form factor for solving the problem raised, and the experimental study of the sectioning method and the form factor for control of the shape of the laser beam spot was conducted. A PLDbased technical implementation of the method was realized.
\end{abstract}

Keywords: Image processing; Laser systems; PLD(FPGA)

\section{Introduction}

Rapidly growing requirements to modern computational media encourage development of new intelligent methods of information transfer and processing. Rigid requirements to real-time information processing systems force scientists to regularly create and upgrade data transfer systems. Today most internet channels cannot provide data exchange of the required quality between such systems, which, in turn, results in the congestion of those channels and formation of so-called digital bottlenecks. A possible solution of the problem of transfer of large volumes of information is to use a fiber-optic cable, but laying such cable is rather expensive, even on short distances. At the same time, this problem can be solved through application of the laser-based technologies (Khosrofian and Garetz, 1983; Kozhemyako et al., 2003; Basov et al., 1998), one of the most promising models of information transfer for the near future. In this case, for instance, tons of full-length films and virtual worlds could be transferred to any part of the globe in a blink of an eye.

Most satellites transmit information, such as TV programs, by means of microwave radiation, while laserbased information transmission could be hundreds of times faster, which, in turn, will considerably increase the carrying capacity of the channel.

\footnotetext{
* Correspondence: timchen@list.ru

'Department of Telecommunication Technologies and Automatics, State Economy and Technologies University of Transport, 19 Lukashevich Street, Kyiv 03049, Ukraine

Full list of author information is available at the end of the article
}

The laser-based information transmission requires that both a satellite and a receiving unit (RU) were located in a certain position. A position of the RU lens, whose diameter is only several centimeters, must be adjusted to one thousandth degree, otherwise the information transmission will not happen.

During the process of tracing a satellite by a receiving unit, one of the main tasks is to classify a shape of the laser beam spot image, namely, its geometrical characteristics distorted by turbulence and air masses.

To solve a problem of classification and increase an accuracy of determination of the spot object center (Mana et al., 2001; Orlov and Neverova, 2003) by means of the maximal usage of its informational characteristics, frames of the series of laser beam spot images should be classified in order to filter the laser route from significantly noise-distorted images.

Known methods of analysis of the optical object shape, for instance described in (Magnes et al., 2010; Wright et al., 2011; Gannot et al., 2002), due to complexity of operations performed do not allow estimate their shape properly with simple computer aids. That's why image processing using the PLD is particularly relevant in our time (Hartmann et al., 2008; Chien and Chen, 2008).

A goal of the work is to solve a scientific problem of developing a computationally simple and therefore high-performing classification method of surfaces of laser beam spot images with its further modeling using the PLD. 


\section{The sectioning method for the real-time control of the shape of the beam spot surface}

When realizing subsystems of image control and processing in such devises as laser locators or laser transfer system, a number of requirements arises that influence a choice of the method and processing instruments:

1. A unit should be low-weighted, small-sized, and have low energy consumption.

2. Processing should be conducted in the real time operation mode.

All that imposes certain restrictions on the choice of the algorithm and time of its execution. One of the methods allowing to conduct image processing and classification with acceptable parameters is a sectioning method for real-time control of the shape of the beam spot.

\section{The approximate classifying function}

A traditional way of solving a problem of control of the shape of laser beam spots includes formation of the beam spot image $B(x, y)$ on the photosensitive surface of the photo receiver and its further transformation into a signal $U(x, y)$. An amplitude of this signal in each point of the expansion with coordinates $(\mathrm{x}, \mathrm{y})$ corresponds to the intensity in $B(x, y)$, i.e. $U(x, y) \leftrightarrow B(x, y)$. Then the signal $U(x, y)$ is being compared with a reference signal $W(x, y)$ for all points of signal expansion. Signals $U(x, y)$ constitute certain surfaces that may differ by type, relative scale coefficient, vector of the relative coordinate matching, and relative turning angles in the three-dimensional space. That is why comparison of those surfaces must be conducted taking into consideration all possible situations, which requires a huge amount of calculations and is difficult for the realtime realization.
In practically important situations, a required comparison of surfaces $U(x, y)$ and $W(x, y)$ can be realized with a new sectioning method described below. This method includes the following operations:

1) find maximum amplitudes of signals $U(x, y)$ and $W(x, y)$ (Figure 1):

$$
\begin{aligned}
& \mathrm{U}_{\max }=\max U(x, y)=h_{u}, \\
& \mathrm{~W}_{\max }=\max W(x, y)=h_{w} ;
\end{aligned}
$$

2) find values of areas $S_{0,75 u}$ and $S_{0,5 u}$ of sections at levels $0,75 h_{u}$ and $0,5 h_{u}$ respectively for the signal $U(x, y)$, and $S_{0,75 w}$ and $S_{0,5 w}$ at levels $0,75 h_{w}$ and 0,5 $h_{w}$ respectively for signal $W(x, y)$;

3) Calculate approximate values of form factors $\tilde{r}_{v, u}$ and $\tilde{r}_{v, w}$ for signals $U(x, y)$ and $W(x, y)$ respectively;

$$
\begin{aligned}
& r_{v, u} \approx \tilde{r}_{v, u}=r_{s, u}=S_{0,75 u} / S_{0,5 u}, \\
& r_{v, w} \approx \tilde{r}_{v, w}=r_{s, w}=S_{0,75 w} / S_{0,5 w},
\end{aligned}
$$

where $\sim$ is a sign of the approximate value,

$$
\begin{aligned}
& r_{v, u}=V_{0,5 u} / h_{u} S_{0,5 u}, \quad 0<r_{v, u} \leq 1 \\
& r_{v, w}=V_{0,5 w} / h_{w} S_{0,5 w}, \quad 0<r_{v, w} \leq 1
\end{aligned}
$$

$V_{0,5 u}$ and $V_{0,5 w}$ are cumulative values of amplitudes of signals $U(x, y)$ and $W(x, y)$, below levels $0,5 h_{u}$ and $0,5 h_{w}$ respectively;

We will prove that $r_{v} \approx r_{s}$ on the example of the current signal $U(x, y)$ in the following way.

A volume $\widehat{V}_{0,5 u}$ of the figure above the section of its surface $U(x, y)$ at the level $0,5 h_{u}$ may be found by one of the known formulas of the approximate
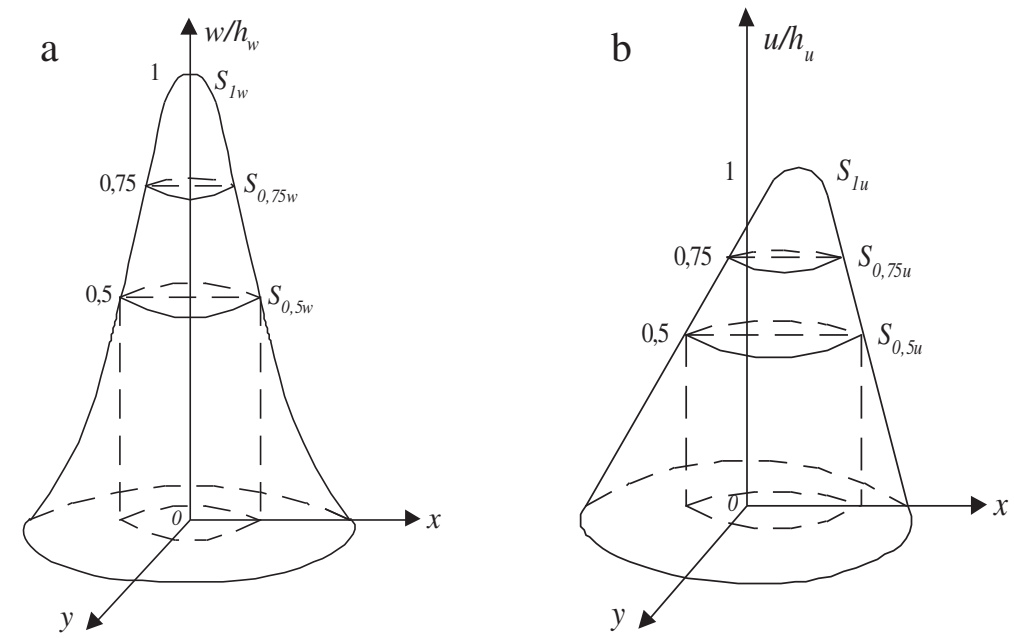

Figure 1 Signals of the laser beam spot image. (a) Reference image and (b) current image. 
calculation of integrals for equidistant nodes, for instance, by Simpson's formula (Kendall, 1989):

$$
\begin{aligned}
\widehat{V}_{0,5 u} & \approx h_{u}\left[S\left(U_{\max }\right)+4 S_{0,75 u}+S_{0,5 u}\right] / 12 \approx \\
& \approx h_{u} S_{0,5 u}\left(4 r_{s, u}+1\right) / 12
\end{aligned}
$$

As $S\left(U_{\max }\right)=S_{1 u}$ is a sectional area of the surface at the level of the maximal amplitude, most often $S_{1 u} \approx 0$.

4) compare form factors $r_{s, u}$ and $r_{s, w}$ (instead of the polyelemental comparison of surfaces).

Using (5), one can easily obtain

$$
V_{0,5 u}=\widehat{V}_{0,5 u}+h_{u} S_{0,5 u} / 2 \approx h_{u} S_{0,5 u}\left(4 r_{s, u}+7\right) / 12
$$

from which follows

$$
\tilde{r}_{v, u} \approx\left(4 r_{s, u}+7\right) / 12
$$

A linear dependence between factors $\tilde{r}_{v}$ and $r_{s}$ makes it possible to use the factpr $r_{s}$ as a characteristic of the form of the respective surface.

Possibilities of classification of surface types with the factor $r_{s}$ are reflected in Table 1. Different values of the factor $r_{s}$ correspond to different types of surfaces of figures, whose examples presented in the table.

An important advantage of the factor $r_{s}$ for some types of surfaces is its independence from the scale coefficient, shift and orientation of the respective surface.

The factor $4 r_{s}$ characterizes a generalized surface convexity: if $4 r_{s}>1$, the surface is convex; if $4 r_{s}<1$, the surface is flat in the generalized sense; and if $4 r_{s}=1$, the surface is linear in the generalized sense.

It is important to mention that a surface can be characterized by the area of the effective cross-section $S_{3}$ :

$$
\begin{aligned}
S_{\ni}= & 2\left(V_{0,5}-S_{0,5} \cdot h \cdot 0,5\right) / h \approx \\
& \approx 2\left(S_{0,5} \tilde{r}_{v} h-S_{0,5} \cdot h \cdot 0,5\right) / h=2 S_{0,5}\left(\tilde{r}_{v}-0,5\right)
\end{aligned}
$$

The sectioning method may be used for a laser beam signal sample, and the shape coefficient may be used as a sample parameter. This method will be used to determine laser beam centers.

$$
S_{\ni} \approx \tilde{S}_{\ni}=\left\{\begin{array}{c}
\left(4 S_{0,75}+S_{0,5}\right) / 6 \text { if } S_{1} \approx 0, \\
\left(S_{1}+4 S_{0,75}+S_{0,5}\right) / 6 \text { if } S_{1} \neq 0,
\end{array}\right.
$$

where $S_{1}$ is a sectional area of the signal on its maximal level.

\section{The algorithm of control of the laser beam spot shape and experimental results}

An algorithm of the method for a frame of $128 * 128$ decomposition elements (DE further on) is the following:

1) Find a point with the maximum brightness $W_{\max }$ on the image;
2) Find values of brightness:

$$
\mathrm{W}_{0.75}=\mathrm{W}_{\max } * 0,75 \text { and } \mathrm{W}_{0,5=0,5} * \mathrm{~W}_{\max } .
$$

3) Find areas of surfaces $S_{0.75}$ and $S_{0.5}$ :

$$
\begin{aligned}
& S_{0.75}=\sum_{x=0}^{N-1} \sum_{y=0}^{N-1}\left\{\begin{array}{c}
S_{0.75}+1, w\left(f(x, y) \geq w_{0.75},\right. \\
S_{0.75}, w\left(f(x, y)<w_{0.75}\right.
\end{array}\right. \\
& S_{0.5}=\sum_{x=0}^{N-1} \sum_{y=0}^{N-1}\left\{\begin{array}{c}
S_{0.5}+1, w\left(f(x, y) \geq w_{0.5}\right. \\
S_{0.5}, w\left(f(x, y)<w_{0.5}\right.
\end{array},\right.
\end{aligned}
$$

where $N$ is a number of decomposition elements in the frame line (column) equal to $128, \mathrm{~W}(\mathrm{f}(\mathrm{x}, \mathrm{y}))$ is a brightness value of the point.

4) Then we will find form factors $r$ :

$$
r=\frac{S 0.75}{S 0.5}
$$

It was found out experimentally, that an image is "good" if $0.7<r<0.8$; if not, go to step 1 .

5) Find image centers (González and Woods, 2003):

$$
\begin{aligned}
& x=\frac{1}{M} \sum_{x=0}^{N-1} \sum_{y=0}^{N-1} w(f(x, y)) * x, \\
& y=\frac{1}{M} \sum_{x=0}^{N-1} \sum_{y=0}^{N-1} w(f(x, y)) * y \\
& M=\sum_{x=0}^{N-1} \sum_{y=0}^{N-1} w(f(x, y))
\end{aligned}
$$

where $\mathrm{x}, \mathrm{y}$ are values of $\mathrm{x}$ and $\mathrm{y}$ coordinates respectively; $\mathrm{w}(\mathrm{f}(\mathrm{x}, \mathrm{y}))$ is the point brightness value; $\mathrm{M}$ is the image "mass" (a sum total of brightnesses of all points of the image).

The following operations are performed during the investigation of the laser beam route:

An initial sample is formed of $10 \%$ laser beam spot images. During its formation, tunnel boundaries are determined with the enumerative technique. After that, sampling of laser beam spot images is conducted, and a group of "good" images is determined (Figure 2).

15 laser beam routes were studied (Basov et al., 1998), and the following results were obtained (presented for two routes):

Tunnel boundaries $\mathrm{a}=0.77, \mathrm{~b}=0.7$. Those values are determined by analyzing a training sample with a method of optimization immediately before running the algorithm.

Difference between the maximum and minimum values of coordinates is 1.147 .

\section{Modeling of the sectioning method for classification of laser beam spots based on the PLD}

The algorithm described in The algorithm of control of the laser beam spot shape and experimental results 
Table 1 Classification of surface types by coefficient $r$

\section{Shape of the longitudinal}

section

1. Prism, cylinder

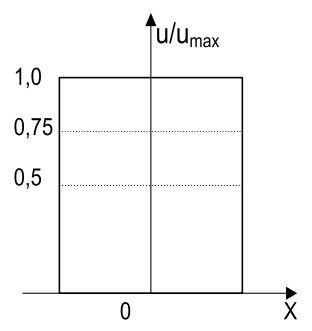

2. Pyramid, cone

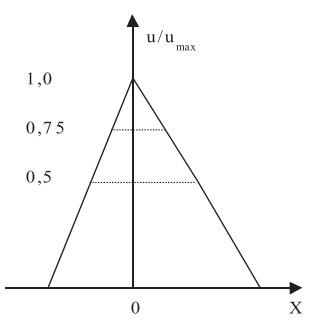

1

$11 / 12 \approx 0.92$

1

$1 / 12 \approx 0.083$
3. Hemisphere

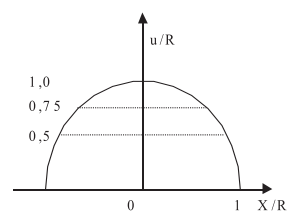

$1 / 4=0.25$

$7 / 12 \approx 0.58$

$7 / 9 \approx 0.78$

0

4. Hemisphere with a base

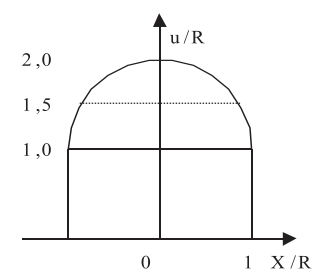

$3 / 4=0.75$

$5 / 6 \approx 0.83$

$5 / 6 \approx 0.83$

0

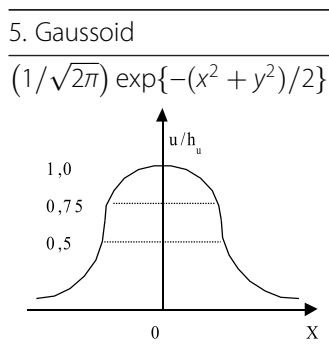

$\ln (4 / 3) / \ln 2 \approx$

0.415
$8.66 / 12 \approx$

0.722
$1 / 2 \ln \approx$

0.721 $\approx 0$ 

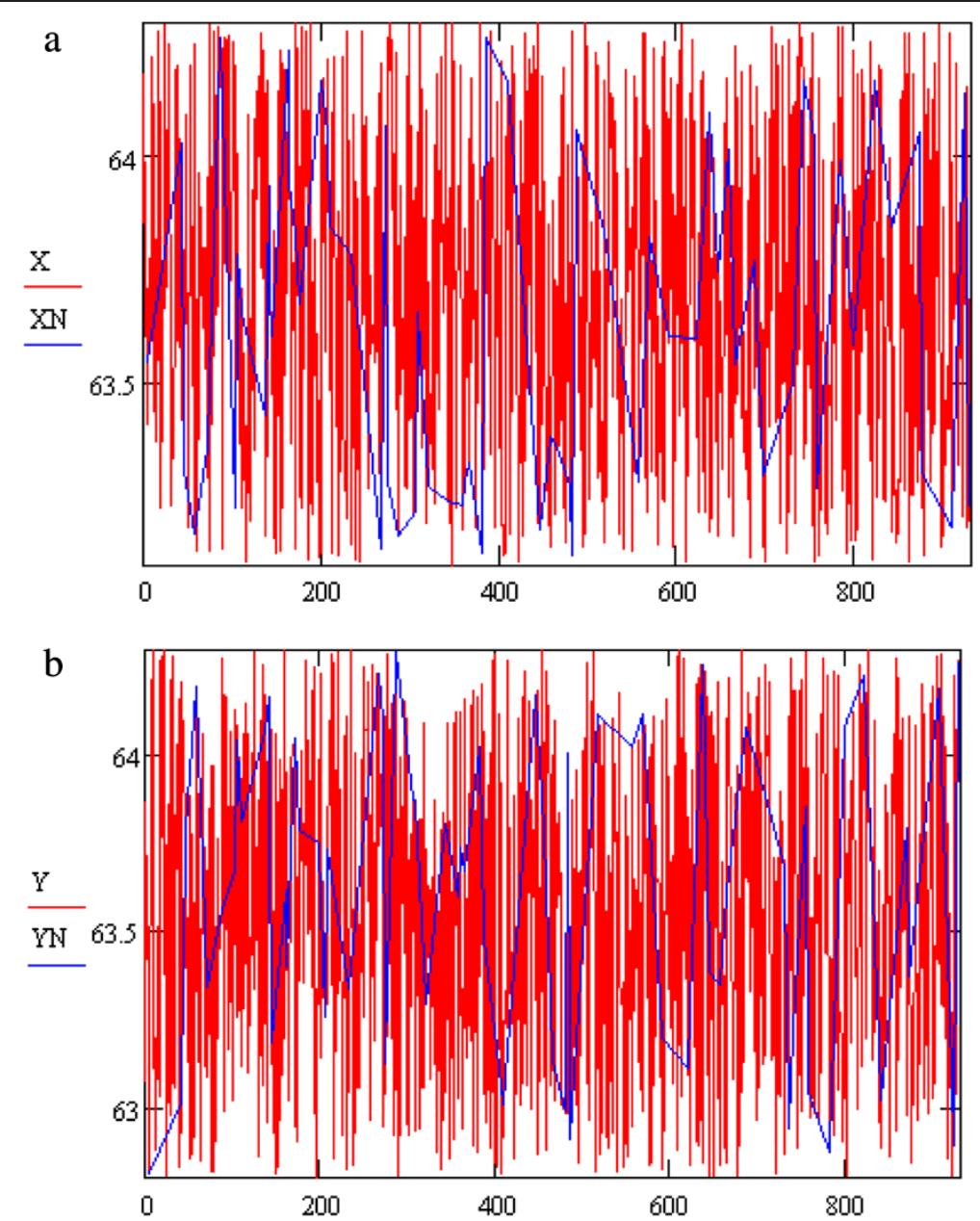

Figure 2 A distribution of coordinates of laser beam centers. (a) $X$-value and (b) $Y$ - value of centers (where $X$ and $Y$ are coordinates of the whole laser beam route respectively, XN, YN are coordinates of spot centers after sampling).

section is relatively simple but has high performance, which allows to use it in add-in systems. The algorithm was used for the first time for this problem based on the Texas Instruments signal processor TMS320C5510 (Timchenko and Kutaev, 2002) with clock frequency of $200 \mathrm{MHz}$; it takes $10 \mathrm{~ms}$ to process one image. About 130 commands are used per one decomposition element.

At the same time, the following drawbacks were revealed in the process of operation: a high enough frequency presents increased requirements to the electromagnetic compatibility and a level of unit realization; almost all processor time is used for image processing, which does not allow to use the processor for other operations.

In addition, at present, video matrixes with higher resolution may be used to increase image refinement. Processing time of one image with the same process for work, for instance, with a matrix of 256 by 256 decomposition elements, may be calculated by the formula:

$$
\tau=\frac{1}{F} * \sum_{i=1}^{k} n_{i}
$$

where $F$ is the processor frequency of $200 \mathrm{MHz}, k=130$ is a number of commands per decomposition element, $n$ is a number of decomposition elements in a frame (256 by $256 \mathrm{DE}=65536 \mathrm{DE}$ ), i.e. processing of one frame takes:

$$
\tau=\frac{1}{2 * 10^{8}} * 130 * 65536=42,5 * 10^{-2} \text { MS. }
$$

Processing time $\tau$ indicates that the processor frequency is not sufficient for the real-time processing and requires a four times more powerful processor. And in case of an even bigger matrix, a need in increased performance grows exponentially. 


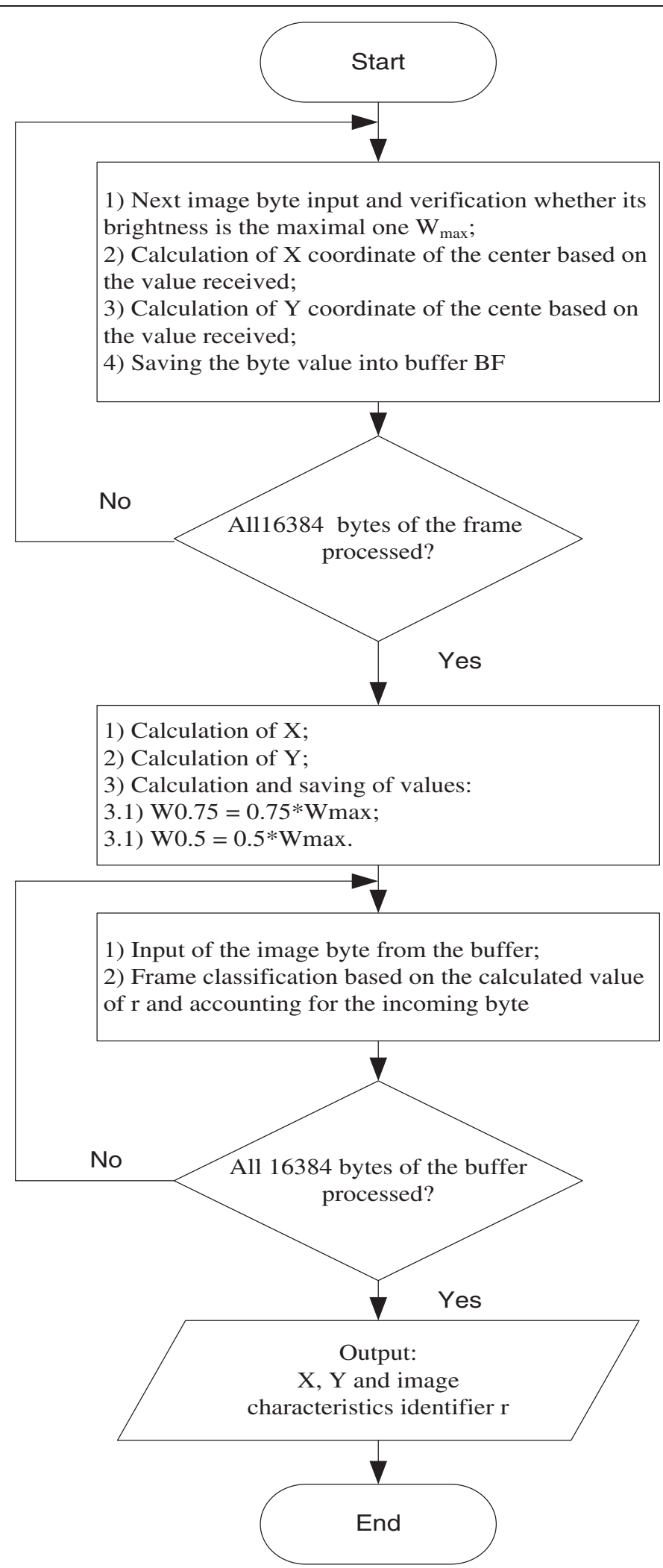

Figure 3 Flowchart of the parallel algorithm of image processing. 


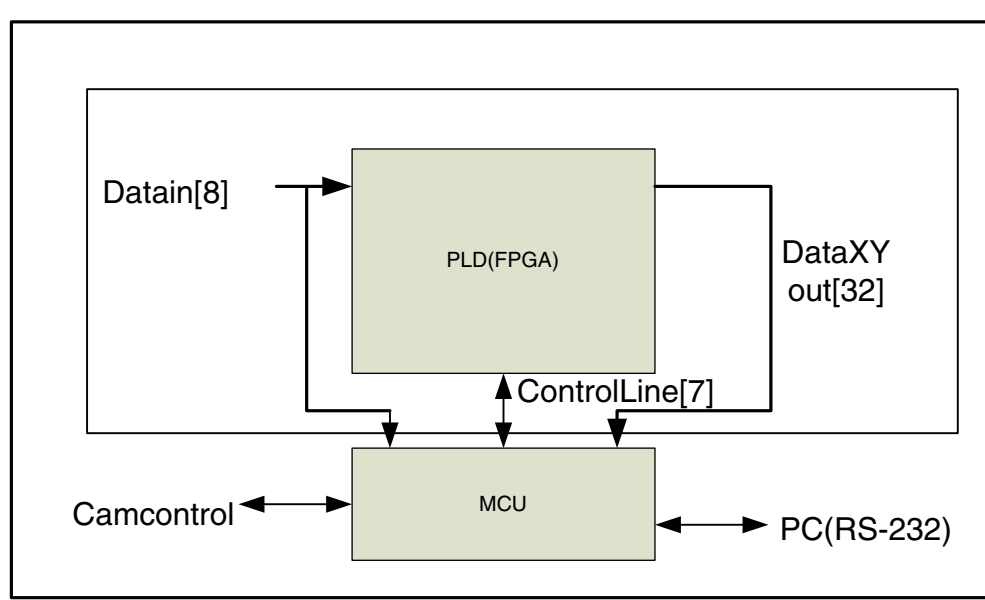

Where:

MCU - microcontroller unit;

PLD(FPGA) - FPGA-type programming logic device;

Datain[8] - 8-digit input data bus (pixel brightness);

Cam control - camera control bus;

ControlLine[7] - PLD control bus;

DataXYout[32] - 32-digitoutput bus for X and Y coordinates;

PC (RS-232) - port for communication with the PC and other system's components (the UART interface is used

as an example)

Figure 4 Block diagram of the image processing and classification device.

\section{Development of a parallel algorithm for the sectioning} method

The following conclusions can be made after a more detailed study of the algorithm:

1. Most operations used in the algorithm are simple mathematical operations (multiplication and addition, comparison and counting).
2. Some formulas use the same variables (for instance, $w(f(x, y))$ in formulas 1 and 3), which provides an opportunity of their sharing.

Hence, a conclusion can be made about a possibility to use the computational hardware developed especially for the algorithm realized, which would allow building the parallel computational structures. The

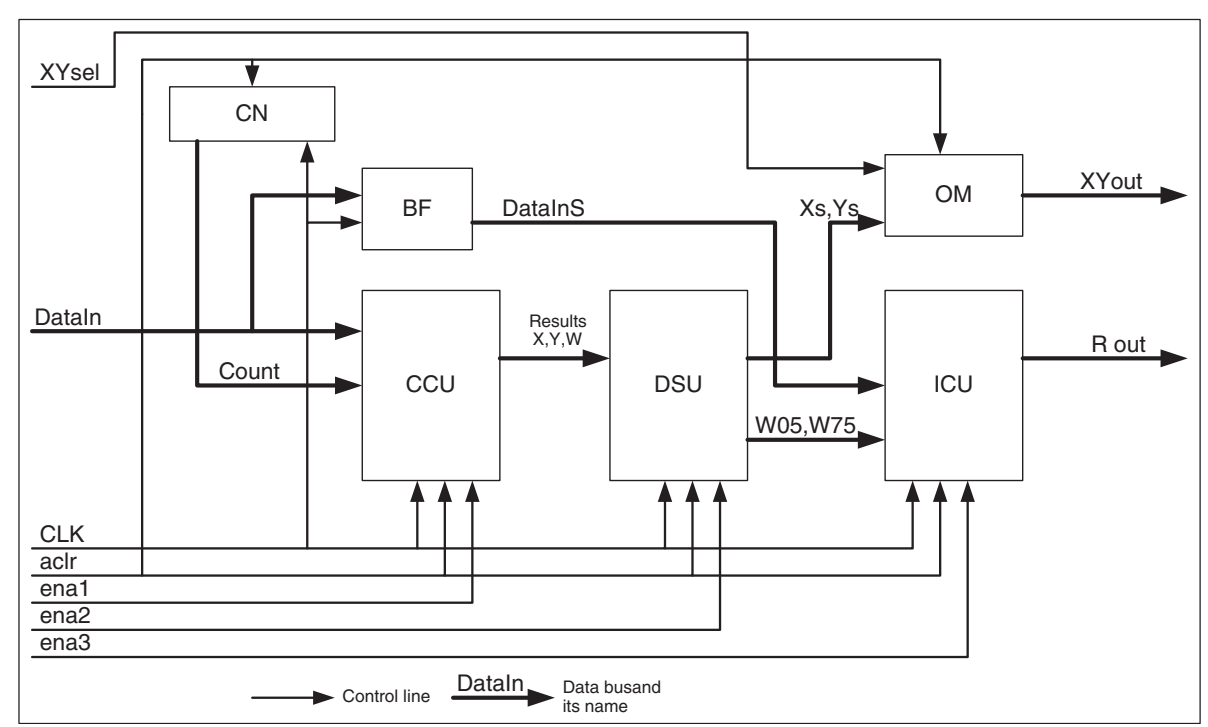

Figure 5 Block diagram of the PLD module. 
Table 2 Buses of the PLD module

\begin{tabular}{lcl}
\hline Name & Direction & Comment \\
\hline Aclr & In & Asynchronous clear input; \\
\hline CLK & In & Clock input; \\
\hline Ena1 & In & CCU clock enable input; \\
\hline Ena2 & In & DSU clock enable; \\
\hline Ena3 & In & ICU clock enable; \\
\hline Rpin & Out & "Good"/"bad" image indication output \\
\hline Datain[8] & In & Input data bus; \\
\hline Dataxy[32] & Out & X and Y output data bus; \\
\hline XYsel & In & Selection of the output coordinate: «0»- X, «1»-Y;
\end{tabular}

most flexible tool for that are the programming logic devices (PLDs).

A parallel algorithm of position determination and classification of laser beam spot images is presented in Figure 3.

Though the flowchart is consequential, operations in each computational unit are performed simultaneously, which results in time-saving in performing operations of the same type.

In the process of development of this algorithm, an order of some operations was changed in comparison with the consequential algorithm. For instance, according to step 4 of the algorithm, a form factor is calculated, and then on its basis a decision about the position computation is made. Instead, in the parallel algorithm, the position is calculated simultaneously with finding a decomposition element with the maximum brightness $\mathrm{W}_{\max }$. This is explained by the fact that in the PLD, unlike the traditional programming, algorithm elements occupy the circuit space regardless of whether they will be performed at a certain stage or not
(Wakerly, 1999). In addition, this technique allows to decrease a number of iterations performed over one image frame.

For the simplicity of implementation, the algorithm implies a PLC-based computational part of the schematic, and the computational process is controlled by means of an external controller or an embedded controller kernel of PLD (Altera, 2011). A block diagram of the device is presented in Figure 4.

In this schematic, a microcontroller unit is controlling a camera, the PLD and PC communication, and the PLD is occupied with calculations of coordinates and image characteristics.

\section{The PLD-based modeling of the parallel algorithm of the sectioning method}

According to the parallel algorithm, a schematic of the image control and processing unit should meet the following requirements:

1. be friendly to upgrades depending on the video matrix used;

2. have a module structure for controlling individual units and maximally reduce the operation time.

Those requirements are satisfied by a schematic presented in Figure 5 and developed on the basis of the Quartus II 10.1 software by Altera, one of the world leaders in PLD production. The software uses a graphics builder and VHDL hardware description language (Ashenden, 1995) in respect to possessing of 128 by 128 DE frames. At the same time, usage of registers with excessive

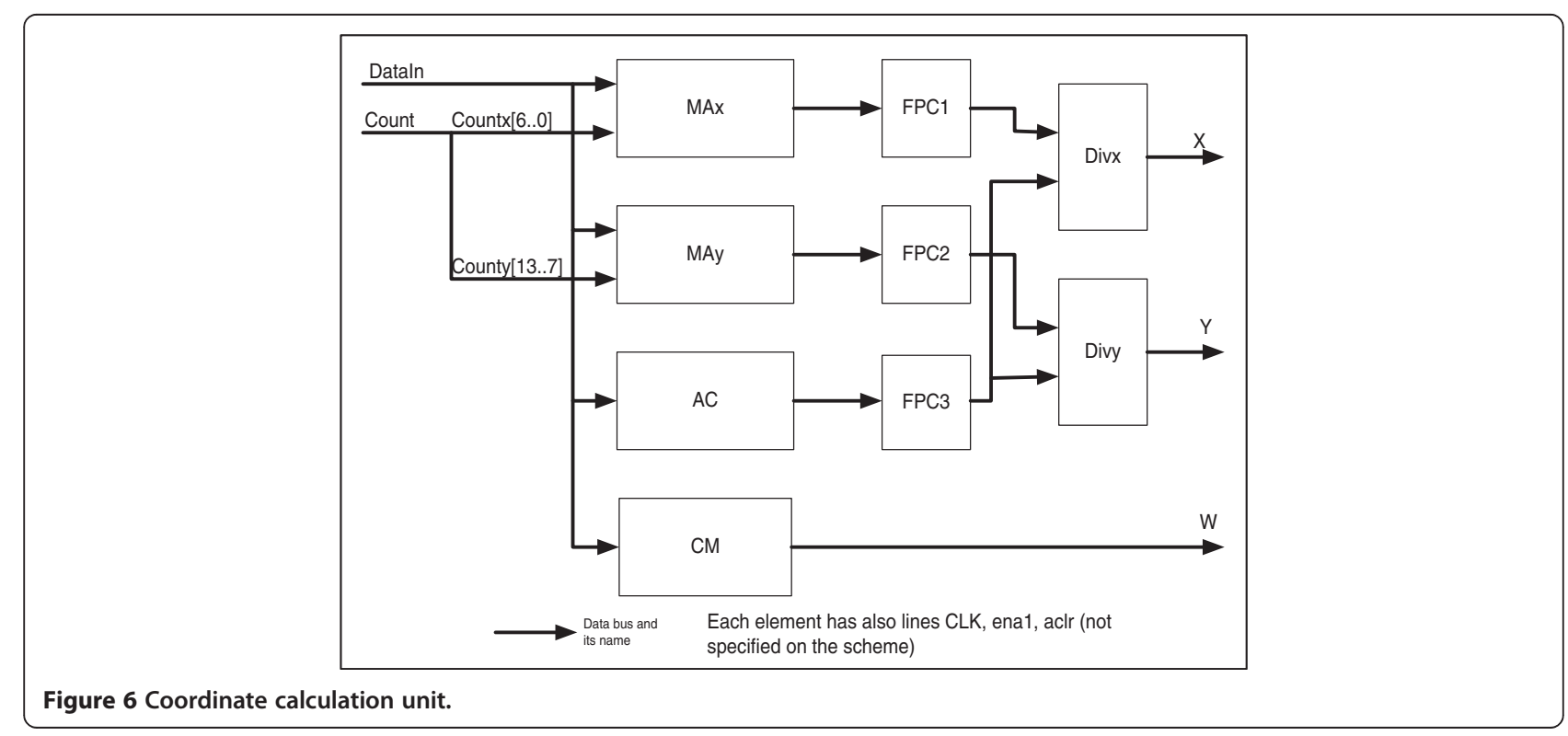


Table 3 Signals of the CCU

\begin{tabular}{lcl}
\hline Name & Direction & Comment \\
\hline Aclr & In & Asynchronous clear input; \\
\hline CLK & In & Clock input; \\
\hline Ena1 & In & Clock enable input \\
\hline Dataln & In & Input data bus; \\
\hline Countx[6..0] & In & Counter input bus for $X$ \\
\hline County[13..7] & In & Counter input bus for $Y$ \\
\hline$X[32]$ & Out & Output of $X$ value; \\
\hline Y[32] & Out & Output of $Y$ value; \\
\hline W[8] & Out & Output of $W$ value; \\
\hline
\end{tabular}

capacity allows to apply it to bigger matrixes as well without significant alterations.

The device consists of the following blocks:

The coordinate calculation unit (CCU) calculates values of $\mathrm{X}$ and $\mathrm{Y}$ and the maximal brightness $\mathrm{W}_{\text {max }}$; the counter $(\mathrm{CN})$ is used to submit input byte coordinates (its number) to the CCU; the data storage unit (DSU) stores intermediate values of $\mathrm{X}$ and $\mathrm{Y}$ and values of $\mathrm{W}_{0,5}$ (W0.5) and $\mathrm{W}_{0,75}$ (W0.75), the image classification unit (ICU) determines a form factor $r$ (classifies images as "good" or "bad"); the 16384-byte circular buffer (BF) is used to store frame data during the circuit operation; the output multiplexor $(\mathrm{OM})$ is an asynchronous multiplexor enabling a serial output of $\mathrm{X}$ or $\mathrm{Y}$ coordinates.

External buses of the circuit are presented in Table 2:

The circuit works in the following way:

After a signal "1" arrives at the bus aclr, all registers are cleared, and a circuit is converted into an initial state. After that, a signal of logical "1" is sent to the bus input ena1 (it also gets to the BF storage input); a data byte is delivered to the input Datain; then a clock pulse passes. A value of the counter $\mathrm{CN}$ increases at the clock pulse edge, and the data is recorded in the buffer BF. The operation is repeated for 16384 clock pulses until the processing of the frame with the dimensionality of 128 by $128 \mathrm{DE}$ is completed, and values of $\mathrm{X}$ and $\mathrm{Y}$ coordinates and of the maximal brightness $\mathrm{W}$ appear at the CCU output therewith.

At the next step, a signal of logical ' 1 ' is fed to the ena2 input, and a signal of logical ' 0 ' is fed to the ena1 input; then a clock signal CLK arrives; as a result, data for $\mathrm{X}, \mathrm{Y}$ and $\mathrm{W}$ are recorded for storage in the DSU.

The third step includes nulling of ena 1 and ena 2 and feeding a signal of logical ' 1 ' to the ena3 input; then 16384 clock pulses arrive to the CLK input. As a result, comparison of brightness of the individual byte with values of half (W05) and $3 / 4$ (W075) of maximal brightness takes place in the ICU, and areas of respective sections are determined. If their dividend (form factor $r$ ) falls within permissible limits, the image is considered "good". To save time during this unit's operation, values of X and Y may be obtained from the output DataXY using a control output $X Y$ sel (logic '0' signal means value $\mathrm{X}$, ' 1 ' - value Y respectively).

After that, an asynchronous reset should be performed, and the system is ready to process a new image. Therefore, the image processing time is 32770 clock pulses.

Let us consider individual units of the schematic in more details. The coordinate calculation unit CCU is presented in Figure 6, and functions of its inputs are presented in Table 3.

The unit consists of the following elements:

MAx, MAy - multiplier-accumulators for X and Y coordinates respectively, they store all values for $\mathrm{x}$ and $\mathrm{y}$; $\mathrm{AC}$ - accumulator of weight values $(\Sigma \mathrm{W})$; $\mathrm{CM}-\mathrm{com}-$ parator and 8-digit register - a circuit of selection and storage of the maximal brightness point; FPC1-3 (code converter) - converters of the 32-digit unsigned integer into a 32-digit floating-point number; DIVx, DIVy (X and $\mathrm{Y}$ dividers respectively) - 32-digit dividers of floatingpoint numbers.

After a general reset, logical " 0 " is set up in registers of all elements, then the byte appears at the input DataIn, and after the clock pulse is received, its value is sent to the inputs Max, where it is multiplied by the counter value. After that, the result obtained is accumulated in the accumulator.

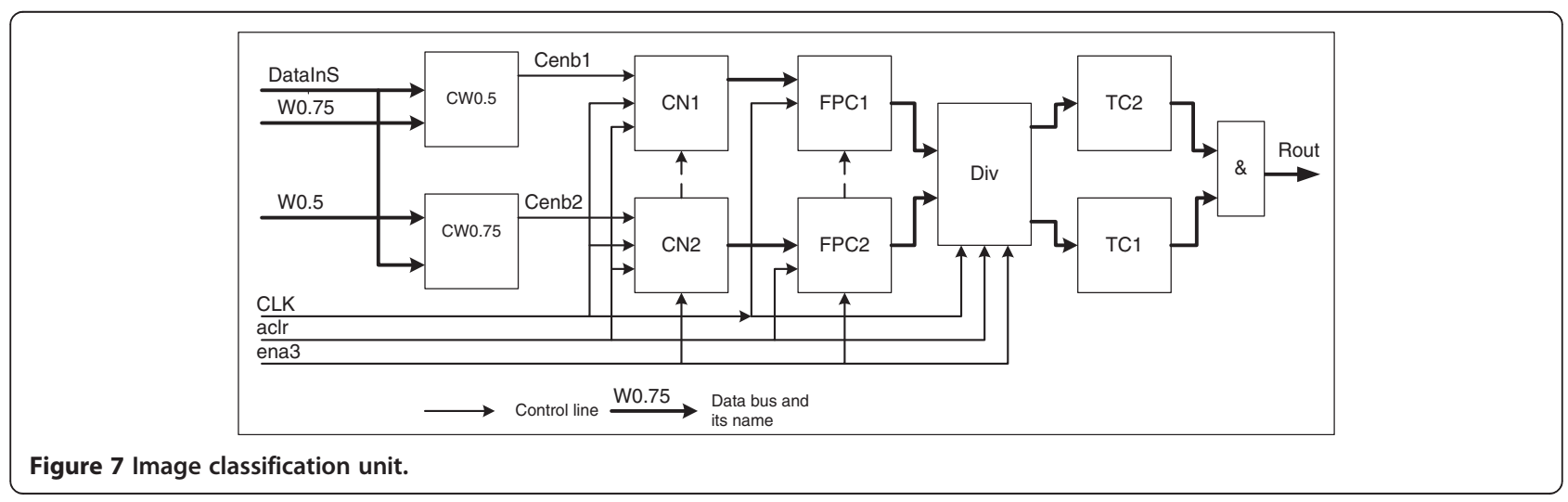


Table 4 Signals of the ICU

\begin{tabular}{lll}
\hline Name & Direction & Comment \\
\hline Aclr & In & Asynchronous clear input; \\
\hline CLK & In & Clock input; \\
\hline Ena3 & In & Clock enable input; \\
\hline DatalnS[8] & In & Input data bus (from buffer) \\
\hline W0.5 & In & Input bus W05; \\
\hline W0.75 & In & Input bus W075; \\
\hline Rout & Out & "Good"/"bad" image indication output \\
\hline
\end{tabular}

Data for Y coordinate are processed similarly, and for W data a simple addition with accumulation is realized. Then obtained sums arrive to dividers Divx, Divy through the code converter (FPC). At the same time, the CM circuit compares value $\mathrm{W}$ of the previous byte with its current value, and if the new value is bigger, it is being registered in the register. As we stated above, one frame of 128 by 128 $\mathrm{DE}$ requires 16384 clock pulses. As a result, values of $\mathrm{X}, \mathrm{Y}$ and W appear on the outputs.

The data storage unit (DSU) is a simple register where intermediate data is stored.

The image classification unit (ICU) is the most complicated element (Figure 7). This unit is designed for calculation of the form factor $r$, i.e. for image classification into "good", with $r=1$, and "bad", with $r=0$ respectively. The unit consists of the following elements and connections (Table 4).

The CW0.5, CW0.75 are comparators that compare incoming frame byte with respective values of brightness; counters $\mathrm{CN} 1, \mathrm{CN} 2$ count a number of points above a respective brightness threshold; code converters FPC1, FPC2 transform an integer into a floating-point number;
Div is a divider of floating-point numbers received from $\mathrm{CN} 1$ and $\mathrm{CN} 2$; threshold comparators TC1 and TC2 compare values received from the Div with preset constants (TC1 for $\mathrm{r}=0.7$ and $\mathrm{TC} 2$ for $\mathrm{r}=0.8$ ).

The circuit works in the following way:

When an input byte arrives, comparators CW0.5 and CW0.75 produce authorization signals for their counters. On arrival of the clock pulse, counters increase their indications by one subject to the authorizing signal of the comparator (CW0.5, CW0.75). This operation is repeated for 16384 times for all frame decomposition elements. Then counter indications are transformed into floating-points numbers, and an operation of their division is performed in the Div. The number obtained is compared in the threshold comparators TC1 and TC2 with constants. If this number is within the limits, logical " 1 " will appear at their outputs, which send a signal of logical " 1 " through the element " $\&$ " to the output, therefore signaling about a "good" image.

The circuit was modeled in the media ModelSim Altera 6.6. on the basis of the Cyclone II microchip of EP2C20Q240C8 type. As a result, the following results were obtained:

Number of logic elements - 2,454/18,752 (13\%).

Number of pins used -47 .

$f_{\max }-112 \mathrm{MHz}$.

Therefore, a microchip with a lower capacity may be used for the real device.

A timing diagram of the PLD unit is presented in Figure 8.

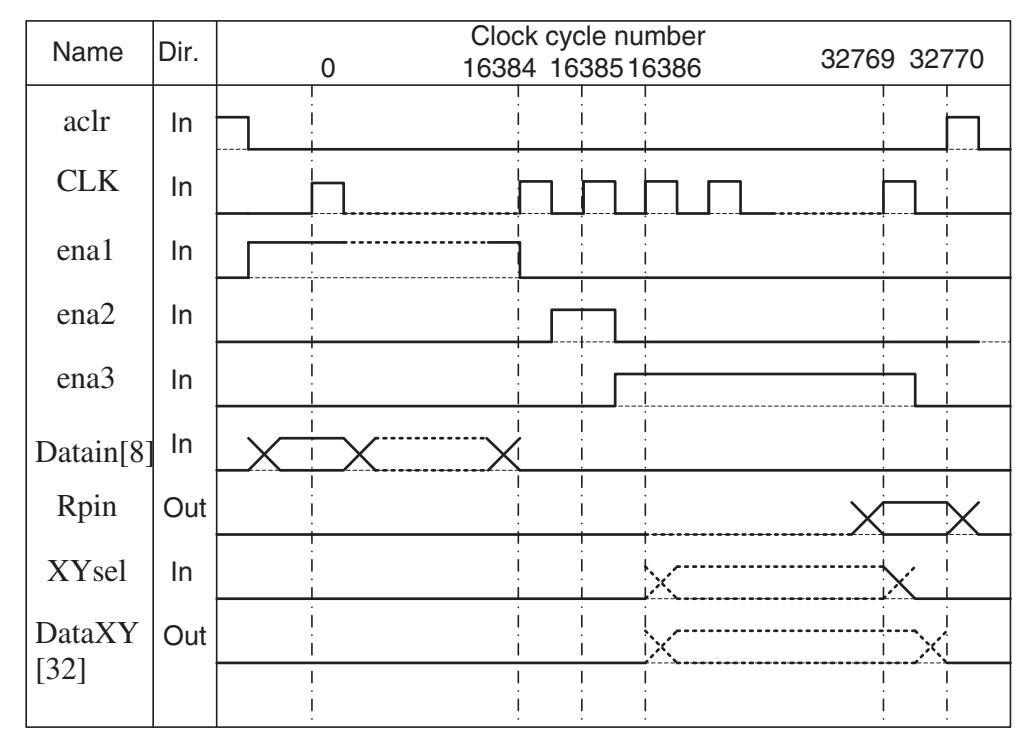

Figure 8 PLD timing diagram. 


\section{Discussion of the obtained results}

Experiments demonstrate that due to various destabilizing factors, coordinates of energy centers of laser beam spots cannot be measured accurately; however, accuracy can be substantially increased by using a calculation of the form factor of images with their further classification into "good" and "bad".

According to the suggested sectioning method, the comparison of surfaces is reduced to the comparison of their form factors and does not require their comparison by element with consideration of all cases of difference in their types, scale coefficient, relative shift and turn in space. It is important to mention that equality of form factors of surface, in the general case, allows to attribute them to the same generalized surface type or to approximate surfaces with that type.

Obviously, the sectioning method can easily be extended to the case of the increased number of equidistant sections of the surface, of changes in values of section levels, changes of weight coefficients in areas of sections. In this case, it also makes sense to use a suitable formula of approximate calculation of integrals.

The sectioning method discussed is also promising for the usage in problems of real-time image classification and archiving. An important advantage of this method is simplicity of its realization in terms of both software and hardware.

A conclusion can be made from the diagram (Figure 8) that the schematic is completely static, and that its operation requires $(2 \mathrm{~N}+2)$ clock pulses to process an image (where $\mathrm{N}$ is a number of decomposition elements), knowing that the initial circuit uses $\tau=10 \mathrm{~ms}$ per frame. A required PLD frequency may be calculated:

$$
\begin{aligned}
& f=\frac{(2 N+2)}{\tau} ; \\
& f=\frac{(2 * 16834+2)}{0.01}=3.27 \mathrm{MHz},
\end{aligned}
$$

As the maximum chip frequency is $100 \mathrm{MHz}$, it can be used for matrixes of up to $512 * 512 \mathrm{DE}$ without changing a chip for a more expensive and high-performance one.

Therefore, this schematic may be used to improve characteristics of laser transfer systems of various types. A flexibility of PLD programming allows to create such subsystem with a single circuit solution, and characteristics of image processing may be changed through reprogramming the PLD for a target transfer system.

In addition, a relatively small space used by the circuit developed in relation to the capacity of PLD logic cells allows making a conclusion that a research should be conducted on embedding one of broadly available chip architectures. This would allow to remove the microcontroller (Figure 4) from the circuit, thus making it even simpler.
The circuit developed may also serve as a basis for developing subsystems of forecasting characteristics of spot images, which becomes more and more needed with a growth in speed and distance between the transfer systems.

\section{Conclusions}

1. A sectioning method for classification of laser beam spots was developed, which does not require performing time-consuming calculations.

2. A sectioning method-based parallel algorithm developed meets conditions by a number and complexity of operations.

3. The image processing and classification device allows to release the main processor from making one-type operations.

4. The working frequency of the circuit in its basic is 40 times lower than with the use of the digital transfer system, which allows to reduce requirements by the electromagnetic compatibility.

5. The processing device design is modular, which makes it possible to use it for matrixes with higher resolution.

Competing interests

The authors declare that they have no competing interests.

\section{Authors' contributions}

VG and YK conducted field research of laser tracks, and took a part in development of the sections method. LT and NK participated in the design of the study, carried out the statistical analysis and drafted the manuscript. MP has developed a parallel algorithm and it's implementation on PLD. All authors read and approved the final manuscript.

\section{Author details}

${ }^{1}$ Department of Telecommunication Technologies and Automatics, State Economy and Technologies University of Transport, 19 Lukashevich Street, Kyiv 03049, Ukraine. ${ }^{2}$ KIA Systems, Moscow, Russia.

Received: 27 June 2013 Accepted: 10 December 2013

Published: 27 December 2013

\section{References}

Altera (2011) Nios II Processor Reference. http://www.altera.com/literature/hb/ nios2/n2cpu nii5v1.pdf

Ashenden PJ (1995) The Designer's Guide to VHDL, 1st edn. Morgan Kaufmann, USA, p 668

Basov NG, Zemskov EM, Kutaev YF et al (1998) Laser control of near earth space and possbilities for removal of space debris from orbit with explosive photo-dissociation lasers with phase conjugation. In: Proceedings of GCL/HPL 98, SPIE Symposium, St. Petersburg, Russia, Vol. 3574. pp 219-228

Chien S-Y, Chen L-G (2008) Reconfigurable morphological image processing. Journal of SPS 56(2-3):155-165

Gannot I, Ben-David M, Inberg A, Waynant RW (2002) Beam shape analysis of waveguide delivered infrared lasers. SPIE, Opt Eng 41:244

González RC, Woods RE (2003) Digital Image Processing Using MATLAB. Pearson Prentice Hall, Upper Saddle River, NJ, p 609

Hartmann M, Pantazis V, Vander Aa T, Berekovic M, Hochberger C (2008) Still image processing on coarse-grained reconfigurable array architectures. Journal of SPS 60(2):225-237

Kendall E, Atkinson (1989) An Introduction to Numerical Analysis, 2nd edn. John Wiley \& Sons, Canada, p 256

Khosrofian JM, Garetz BA (1983) Measurement of a Gaussian laser beam diameter through the direct inversion of knife-edge data. Appl Opt 22:3406-3410 
Kozhemyako VP, Timchenko LI, Poplavskyy AA et al (2003) Analysis of the methodological approaches in connection with the problem solving of extrapolation of object trajectory. SPIE Symposium, USA 5175:222-236

Magnes J, Kinneberg M, Khakurel R, Melikechi N (2010) Opto-mechanical shape analysis using group theory. Appl Opt 49(22):4188-4192

Mana G, Massa E, Rovera A (2001) Accuracy of laser beam center and width calculations. Appl Optics 40(9):1378-1385

Orlov DA, Neverova EA (2003) Determination of the position of the center of a laser beam when the dynamic range of the matrix receiver is exceeded. Meas Tech 53(10):1140-1146

Timchenko LI, Kutaev YF (2002) Method for training of a parallel-hierarchical network, based on population coding for processing of extended laser paths images. Proc SPIE 4790:465-479

Wakerly JF (1999) Digital Design: Principles and Practices. Prentice Hall, Upper Saddle River, NJ, p 949

Wright MW, Wilson KE, Kovalik J, Biswas A, Roberts WT (2011) A bidirectional low earth orbit-to-ground optical link experiment. SPIE Newsroom:3, doi:10.1117/2.1201109.003826

doi:10.1186/2193-1801-2-692

Cite this article as: Tymchenko et al:: Modeling of the high-performance PLD-based sectioning method for classification of the shape of optical object images. SpringerPlus 2013 2:692.

\section{Submit your manuscript to a SpringerOpen ${ }^{\circ}$ journal and benefit from:}

- Convenient online submission

- Rigorous peer review

- Immediate publication on acceptance

- Open access: articles freely available online

- High visibility within the field

- Retaining the copyright to your article

Submit your next manuscript at $>$ springeropen.com 